\title{
Aplicaciones de teCnOlogías de INFORMACIÓN: ADOPCIÓN Y DIFUSIÓN DEL COMERCIO ELECTRÓNICO EN GRANDES EMPRESAS DE IZTAPALAPA
}

\section{Regina Leal Güemez}

\section{Salvador T. Porras Duarte ${ }^{1}$}

\section{Resumen}

El comercio electrónico para las empresas sin duda representa una alternativa de negocio muy atractiva debido a sus propiedades y ventajas potenciales, sin embargo, también hay una serie de barreras que disuaden su implementación. La investigación en cuanto al uso y aprovechamiento de estas tecnologías por parte de las empresas sigue siendo incipiente, por ello, el objetivo de este trabajo es analizar la difusión de comercio electrónico en un grupo de grandes empresas de la delegación Iztapalapa. Como primera etapa de la investigación se llevó a cabo un análisis cuantitativo y de contenido examinando las páginas web del grupo de empresas bajo estudio, para determinar la adopción y uso del comercio electrónico.

Esta aplicación tecnológica resulta una estrategia natural para empresas dedicadas al comercio, empero no lo es tanto para organizaciones ubicadas en el sector manufacturero o de servicios. Asimismo, la implementación de esta estrategia implica una inversión importante en hardware, software y personal. Por ello creemos que la difusión del comercio electrónico todavía no se ha generalizado en las grandes empresas de la demarcación de Iztapalapa y por lo tanto no han aprovechado sus ventajas.

Palabras clave: tecnologías de información, comercio electrónico, adopción, grandes empresas.

1 Profesores-Investigadores del Departamento de Economía de la Universidad Autónoma Metropolitana, Unidad Iztapalapa. 


\section{Abstract}

Electronic commerce represents a very attractive business option for enterprises because of its properties and potential advantages; however, there is a set of barriers that discourage its implementation. Research related to the use and advantages of these technologies by enterprises continues to be incipient; this paper aims to analyze the diffusion of electronic commerce in a group of large enterprises in the municipality of Iztapalapa, CDMX. The first step of the research consisted in conducting a quantitative and content analysis of the web pages of the enterprises to establish the adoption and use of electronic commerce.

This technological application results a natural strategy for commercial enterprises, but it is not such for organizations in the manufacturing and services sectors. Likewise, the implementation of such a strategy implies an important investment in hardware, software and personnel. For these reasons, we believe that diffusion of electronic commerce has not been generalized in large enterprises in Iztapalapa, losing the benefits of its use.

Keywords: Information technology, Information systems, electronic commerce, large enterprises

\section{Introducción}

El panorama del desarrollo de las tecnologías de información sigue evolucionando constantemente por diferentes razones, entre otras, debido al incremento en el número de usuarios de internet al igual que su acceso a través de banda ancha; cada vez más se utilizan tecnologías móviles para su funcionamiento en internet; hay una gran difusión del uso de redes sociales; los gobiernos con mayor urgencia proveen servicios a través de e-gobierno permitiendo la realización de transacciones en línea, y la nube computacional genera nuevas oportunidades y riesgos (Fredriksson, 2013).

No obstante, el desarrollo de la economía digital en todo el mundo ha sido diferente en velocidad y nivel. Por un lado, la digitalización 
de las actividades económicas y transacciones puede ayudar a derribar barreras que permiten un desarrollado incluyente. Por el otro, también significa una división entre los países preparados para comprometerse y beneficiarse de la economía digital enfrentándose a los riesgos y generando una mayor división entre aquellos países no preparados (UNCTAD, 2017).

De igual forma, el nivel de digitalización de las empresas indica el grado en el cual han adoptado y utilizado las tecnologías de información. La adopción de TI se puede medir a través de indicadores como la disponibilidad de computadoras, el acceso a internet y otras tecnologías de información. Mientras más actividades dentro de la empresa se vuelvan digitales, las compañías requieren tener una presencia en línea que sea visible tanto para los consumidores como para otras empresas (UNCTAD, 2017).

En particular, la Internet ha creado una plataforma tecnológica universal que ha inundado el mercado con aplicaciones dirigidas tanto al uso doméstico como al organizacional, ampliando con ello las posibilidades de las empresas para implementar innovaciones tecnológicas en diferentes niveles y diversas funciones.

Específicamente con relación al comercio electrónico, durante muchos años las empresas utilizaron sistemas patentados para integrar información proveniente de sus sistemas internos y enlazarse con sus clientes y socios comerciales. Estos sistemas resultaban costosos y se basaban en estándares de tecnología que solo algunos podían alcanzar (O’Brien y Marakas, 2007; Stair y Reynolds, 2006). Esta circunstancia también se modificó debido al desarrollo de las tecnologías de información ya que a partir de la creación de la tecnología web WWW (World Wide Web) en 1992, y de la introducción en 1996 del cifrado de seguridad SSL 3.0 (Secure Sockets Layer), se produjo la expansión exponencial del comercio electrónico (Fernández-Portillo, et al., 2015). Actualmente internet se ha convertido en la tecnología universal para comprar y vender bienes, por ello resulta la plataforma natural de elección para el comercio electrónico ya que ofrece a los negocios maneras incluso 
más fáciles de enlazarse con otros negocios e individuos a un menor costo (O’Brien y Marakas, 2007; Stair y Reynolds, 2006).

De hecho, el comercio electrónico se ha desarrollado de tal forma que de ser una actividad pasiva y estática utilizada por las empresas básicamente para mostrar sus catálogos de productos y servicios, se transforma en una herramienta estratégica con capacidades para desarrollar nuevos negocios, expandir las empresas y mantener relaciones más estrechas con clientes y proveedores.

Debido a las características de esta tecnología y al incremento de los usuarios que utilizan los medios electrónicos para buscar y adquirir productos y servicios, las empresas cada vez se ven más en la necesidad de adoptar e implementar el comercio electrónico. Sin embargo, como ya lo mencionamos, el desarrollo de esta aplicación es desigual en el mundo, en particular en México se presenta un lento crecimiento del comercio electrónico a pesar del papel estratégico que puede representar para las organizaciones.

De ahí que el objetivo de este estudio es analizar la difusión del comercio electrónico en grandes empresas ubicadas en la delegación Iztapalapa en la Ciudad de México. El presente trabajo describe en primera instancia el concepto de comercio electrónico, así como sus ventajas y desventajas. A continuación, se exponen las diversas líneas de investigación vinculadas con el estudio del comercio electrónico y se presenta el modelo multi-etapas para la implementación de esta tecnología. Posteriormente se hace una revisión de la situación del comercio electrónico en México para, en el siguiente apartado, explicar la metodología utilizada en esta investigación y continuar con la presentación y análisis de resultados. El documento termina con las conclusiones obtenidas del estudio.

\section{Comercio Electrónico}

Siguiendo a la UNCTAD (2017), se puede definir el comercio electrónico como el uso de equipos de cómputo y redes de telecomunicaciones como herramienta primaria, para realizar las operaciones comerciales básicas y ofrecer los productos y servicios a los clientes a través de 
internet. Implica el uso de múltiples formatos y dispositivos, incluidos el intercambio de datos, la web, el uso de computadoras personales, laptops, tabletas y teléfonos celulares. El comercio electrónico supone llevar a cabo en línea todo el proceso del negocio, el cual incluye desarrollo, mercadotecnia, venta, distribución, servicio y pago por los productos y servicios que se comercialicen en internet (O'Brien y Marakas, 2007; Stair y Reynolds, 2006). En efecto, el comercio electrónico puede incluir mercadotecnia interactiva, órdenes de compra, pagos, apoyo al proceso de compras a través de catálogos, sitios de subastas en la web entre otros (O’Brien y Marakas, 2007; Stair y Reynolds, 2006).

Dependiendo de las transacciones electrónicas a que se hagan referencia, es posible determinar diferentes tipos de comercio electrónico como (O’Brien y Marakas, 2007; Stair y Reynolds, 2006):

- Entre las empresas (business to business B2B) se llevan a cabo ventas de bienes y servicios.

- Entre empresas y clientes (business to consumers B2C) para la venta al menudeo de productos o servicios a compradores individuales.

- Entre consumidor y consumidor (consumer to consumer $C 2 C$ ) se realizan ventas directamente.

- Entre empresa y el sector público (business to government B2G), para que el gobierno pueda operar transacciones comerciales con las empresas.

- Entre consumidores y el sector público (consumer to government $C 2 G$ ), para que igualmente los consumidores puedan realizar por ejemplo pago de servicios al gobierno electrónicamente.

Las ventajas de emplear esta plataforma tecnológica radican en que es universal y fácil de usar, está basada en estándares que todas las organizaciones pueden utilizar, sin importar las características de cada empresa (O'Brien y Marakas, 2007; Stair y Reynolds, 2006). Por ello, puede utilizarse en cualquier contexto donde se requiera el intercambio de documentos entre organizaciones. Las páginas web de las compa- 
ñías están disponibles 24 horas al día, 365 días al año; productos como software, música, videos, libros por ejemplo se pueden distribuir directamente a través de internet. Mientras que los proveedores de otro tipo de productos o servicios pueden difundir en la red información relativa a los mismos como precio, características, opciones, disponibilidad y tiempo de entrega (O'Brien y Marakas, 2007; Stair y Reynolds, 2006).

Dado sus características, el comercio electrónico ha desarrollado a su vez otras áreas como, por ejemplo, la creación de nuevos canales de mercadotecnia y ventas; el acceso interactivo a catálogos de productos y servicios; listas de precios; características específicas y publicidad; soporte técnico ininterrumpido; apoyo al cliente y servicio posventa (Ríos Ruíz, 2014).

Utilizar la tecnología de internet en el proceso de compra-venta genera una serie de ventajas para las empresas, como la posibilidad de reducir sus costos de transacción y tiempos de entrega de algunos bienes -en particular los que son totalmente digitales: software, productos de texto, imágenes o videos-; acelerar el flujo de bienes y de información, mejorar el servicio al cliente y la coordinación entre productores, proveedores y clientes (O’Brien y Marakas, 2007; Stair y Reynolds, 2006).

Además del beneficio en términos de reducción de costos, las empresas también pueden tener una presencia global, disponibilidad ilimitada de horarios, aumento de la competitividad, productos y servicios personalizados, eliminación de intermediarios, oferta de productos de formato digital, nuevas oportunidades de negocio/nuevos productos y servicios, otra forma de mercadeo (medio de publicidad), un mejor conocimiento de sus clientes y una sensible reducción de inventarios (Sojo Obando, 2001).

Mientras que el cliente puede obtener como beneficios al utilizar el comercio electrónico una mayor comodidad de realizar sus compras en línea, economizar en tiempo y recursos, mayor variedad de opciones en productos y precios, suficiente información relativa de las mercancías, disponibilidad de comprar los 365 días del año, trato personalizado basado en compras previas e independencia geográfica (Sojo Obando, 2001). 
Sin embargo, la implementación de esta tecnología puede representar una serie de dificultades para las organizaciones, como una inversión considerable en hardware y software; contratación de personal especializado que atienda los pedidos; el desarrollo de una logística especializada para el envío del producto; un costo financiero de la venta, número de visitas y costo de publicidad (Sojo Obando, 2001).

En la literatura es posible encontrar diversas áreas de investigación relacionadas con el estudio del comercio electrónico. Una vertiente importante se ha enfocado en el análisis de los factores relacionados con la adopción de esta tecnología, como serían aquellos relativos con la organización, factores relacionados con una perspectiva de innovación o aceptación tecnológica, factores técnicos, factores externos vinculados con el medio ambiente (Fernández-Portillo, Antonio, et al., 2015; Frasquet Deltoro, Mollá Descals y Ruiz Molina, 2012; Rodríguez-Ardura, Meseguer Artola y Vilaseca i Requena, 2008; Sepúlveda Robles, et al., 2016). De los cuáles concluyen que algunos factores relacionados con el éxito de la adopción de comercio electrónico son: tamaño de la organización, antigüedad de la empresa, características del producto, grado de innovación, grado de internacionalización, niveles de marketing en internet, la seguridad de las transacciones, el tamaño de la comunidad internauta, la base de infraestructura tecnológica, factores psicológicos y culturales (Fernández-Portillo, Antonio, et al., 2015; Frasquet Deltoro, Mollá Descals y Ruiz Molina, 2012; Rodríguez-Ardura, Meseguer Artola y Vilaseca i Requena, 2008; Sepúlveda Robles, et al., 2016).

También existen estudios que describen cómo ha sido el desarro1lo del comercio electrónico a través del tiempo (Fernández Nodarse, 2013; Guerrero Cuéllar y Rivas Tovar, 2006). Estas investigaciones refieren los primeros modelos que mostraban escaparates virtuales en la red, el objetivo era que la empresa se asegurara de contar con un sitio en la web. Posteriormente se transitó hacia el modelo web 2.0 donde se sitúa al usuario como el centro del negocio y se llevaban a cabo transacciones, compra y venta sobre medios digitales. A continuación se desarrolló la etapa de e-Business en la cual se incluyen todas las aplicaciones y procesos que habilitan a las empresas para poder llevar a cabo 
la transacción comercial de forma digital a lo largo de todo el proceso. Finalmente, se habla de la transición al modelo web 3.0 basado en una web evolucionada con interfase 3D y un amplio empleo de inteligencia artificial (Fernández Nodarse, 2013).

De igual forma, hay investigaciones dirigidas al desarrollo de modelos que expliquen las fases o etapas a través de las cuales las empresas transitan para poder adoptar e implementar el comercio electrónico como parte de la difusión del mismo (Daniel, Wilson y Myers 2002; Guerrero Cuéllar y Rivas Tovar, 2005; Montijano Guardia, et al., 2002; Veciana Vergés y Serrasols i Tarrés (2001). En ese sentido Guerrero Cuéllar y Rivas Tovar (2005, citando a Andrews, 2002) señalan que se pueden establecer tres etapas en el proceso de compra basado en redes de telecomunicación: la primera etapa es de pre-compra donde los clientes tienen acceso a la página web y pueden visualizar su historial de compra y estado; la siguiente etapa corresponde a la de navegar y comprar, en la que los usuarios se conectan con el sitio de la empresa, consultan el catálogo, seleccionan sus productos o servicios y realizan la compra; por último está la etapa integrativa donde la empresa le proporciona al cliente software complementario para integrarlo en el proceso de compra-venta de la empresa y que éste sea un distribuidor de la misma.

Daniel, Wilson y Myers (2002) señalan que las PyMEs adoptan el comercio electrónico por etapas, resultado de la experiencia previa que van acumulando. De tal forma que en cada etapa las empresas desarrollan aplicaciones y actividades vinculadas con esta tecnología que funcionan como base para el siguiente estado. En la primera fase las organizaciones empiezan a desarrollar los primeros servicios de comercio electrónico, es decir, actividades vinculadas con comunicaciones a través de correo electrónico con clientes y proveedores, creando bases de datos de productos y servicios e información para publicitar su propia empresa y marca a través de su sitio web. En la segunda etapa ya se ha implementado la comunicación a través de correo electrónico con clientes y proveedores, ya funciona el sitio web de la empresa como fuente de información de la misma, hay intercambio electrónico de documentos hacia dentro y fuera de la organización. En la siguiente eta- 
pa se empiezan a desarrollar aplicaciones para poder generar y recibir órdenes en línea, es decir la posibilidad de llevar a cabo transacciones digitales. Y en la última etapa se tiene implementado, finalmente, el comercio electrónico, ya que pueden realizar órdenes en línea, proveer servicio posventa y contacto, e inclusive, llevar a cabo el proceso de reclutamiento en línea.

Por su parte Veciana Vergés y Serrasols i Tarrés (2001) y Montijano Guardia et al. (2002) apuntan que las empresas que empiezan a adoptar la tecnología de Internet, incluyendo el comercio electrónico, lo hacen por etapas. De acuerdo con Veciana Vergés y Serrasols i Tarrés (2001), en la primera, la página Web es solamente informativa con el objetivo de tener una presencia en Internet; en la segunda etapa empiezan a trasladar algunos procesos en el sitio Web como por ejemplo catálogos de productos, búsqueda de personal y atención al cliente. Y en la última etapa las empresas cuentan ya con la posibilidad de realizar transacciones en su página.

Para Montijano Guardia et al. (2002) establecen que la presencia de las empresas en la Web puede clasificarse en tres etapas: la web informativa donde las organizaciones utilizan la internet para difundir información de las mismas; el siguiente tipo corresponde a la web transaccional donde se incorporan actividades utilizando el correo electrónico, formularios para dar respuesta a los usuarios y ofrecer sus productos a través de este medio. El último tipo corresponde a la web operacional donde se establece una interconexión con proveedores y clientes para poder realizar intercambio de información electrónica, así como comercializar sus productos a través de internet.

Otra línea de estudio, que está ubicada en una disciplina diferente, representa el marco legal operativo del comercio electrónico corresponde al análisis del marco jurídico y los potenciales actos ilícitos que se cometen al llevarlo a cabo (Pecoy Taque, 2011). Esta rama de estudio cada vez es más importante dado la difusión del uso de comercio electrónico y la propagación de igual forma de ilícitos relacionados con el mismo. 


\section{Modelo multi-etapas para comercio electrónico}

Un sistema de comercio electrónico requiere que las empresas implementen en la plataforma de internet todas las etapas que el cliente experimenta en un ciclo de vida de ventas. Para ello es necesario que el usuario pueda llevar a cabo la búsqueda e identificación de los productos que desea comprar, seleccionar aquellos que le interesen, negociar el precio, escoger los términos y forma de pago y conocer la fecha de entrega; una vez realizado el paso anterior proceder a generar una orden de venta para facturar los productos y realizar el pago; por último, obtener el producto entregado y recibir un servicio posventa (O'Brien y Marakas, 2007; Stair y Reynolds, 2006).

Basado en lo anterior, el modelo multietapas para facturar en internet incluye la búsqueda e identificación, selección y negociación, facturación, entrega de productos o servicios y servicio posventa (O'Brien y Marakas, 2007; Stair y Reynolds, 2006). En ese sentido, resulta esencial que las empresas lleven a cabo un proceso de implementación del sistema que les asegure una operación exitosa. Mismo que debe incluir al menos nueve elementos (O’Brien y Marakas, 2007; Stair y Reynolds, 2006):

- Control de acceso y seguridad de forma que los usuarios tengan confianza en llevar a cabo la transacción de comercio electrónico. Por eso es fundamental incorporar la autentificación de usuarios, un acceso autorizado y refuerzos en las actividades de seguridad del sitio.

- Perfil y personalización para que una vez que el cliente ingresó al sitio el proceso de construcción del perfil pueda llevarse a cabo al recopilar la información del usuario, así como su comportamiento y elecciones en la página Web; con el objetivo de generar electrónicamente su perfil de cliente al definir sus características y preferencias. Este perfil se utiliza para reconocer a cada cliente como un usuario individual y proveer un punto de vista personalizado en el sitio, así como en las recomendaciones de productos. 
- Administración de búsquedas para ayudar a los clientes a encontrar un producto específico o un servicio que ellos quieran evaluar o comprar.

- Administración de contenido para desarrollar, generar, entregar, actualizar y almacenar archivos de datos e información multimedia en los sitios Web.

- Administración de catálogos para generar listas multimedia con la información de productos.

- Administración de pago para que, a través de un carrito de compras, el cliente pueda llevar a cabo su compra, seleccionar la forma de pago y verificarla.

- Administración del flujo de trabajo para asegurar que las transacciones apropiadas, decisiones y actividades de trabajo se realicen, y los datos correctos y documentos se dirijan a los empleados correspondientes, clientes, proveedores y otros miembros del negocio.

- Notificación de eventos dirigidos para responder a diversas actividades tales como primer ingreso al sitio de clientes nuevos, procesos de pago y entrega, y a innumerables actividades para la administración de las relaciones con los clientes y la cadena de suministro.

- Colaboración y comercio son aquellas actividades que soportan la cooperación de acuerdos vitales y servicios comerciales que necesitan los clientes, proveedores y otros participantes que acompañan las transacciones en el comercio electrónico.

\section{Situación del comercio electrónico}

Como ya se mencionó, el desarrollo y difusión de las tecnologías de información han provocado un incremento en la población que se conecta a internet y de igual forma en los negocios que han implementado el comercio electrónico. A nivel internacional, de acuerdo con estadísticas oficiales UNCTAD (2017) relacionadas con los mercados de comercio electrónico, que incluyen tanto negocio a negocio (B2B) y negocio a 
consumidor (B2C), el comercio electrónico global en 2015 alcanzó el monto de \$25,293 billones de dólares. La cantidad de ventas de B2C representaron $\$ 2,904$ billones de dólares, aproximadamente un 10\% del monto global estimado. Mientras que las ventas de B2B alcanzaron $\$ 22,389$ billones de dólares (UNCTAD, 2017). China sigue representando el mayor mercado B2C de comercio electrónico ( $\$ 617$ billones de dólares), seguido por los Estados Unidos (\$612 billones de dólares) (UNCTAD, 2017). Sin embargo, los Estados Unidos, reportan el mayor mercado B2B con $\$ 6,443$ billones de dólares, seguido por Japón $(\$ 2,382$ billones de dólares) (UNCTAD, 2017). Las cifras reportan dentro de los 10 mercados más grandes de comercio electrónico a: Estados Unidos, Japón, China, República de Corea, Alemania, Reino Unidos e Irlanda del Norte, Francia, Canadá, España y Australia. Obviamente, en esa lista no aparece ningún país en vías de desarrollo.

Para el 2014 el mercado de Asia Pacífico se estimó un crecimiento de un 23\%, siendo China (65\%) e Indonesia (71\%) los principales motores del sector (Ríos Ruíz, 2014). De igual manera, Norteamérica y Europa Occidental crecerían en un 12\% (Ríos Ruíz, 2014).

En México las estadísticas indican que la difusión de tecnologías de información ha seguido creciendo, de acuerdo con el INEGI (2016) el número de usuarios de computadora se incrementó de $16.74 \%$ en 2001 a $51.25 \%$ en 2015 ; el número de usuarios de internet pasó del $7.95 \%$ en 2001 a $57.43 \%$ en 2015; y la cantidad de usuarios con teléfono celular que era el 43.91\% en 2009 representó en 2015 el 71.46\%.

De acuerdo con Ríos Ruíz (2014), en México las empresas comenzaron a conectarse en 1994 y el número de entidades que ha implementado el comercio electrónico se ha incrementado paulatinamente, situando a nuestro país en el segundo puesto de los países de América Latina después de Brasil. De acuerdo con la Asociación Mexicana de Internet, se ha quintuplicado el flujo económico producido por el comercio electrónico desde el año 2009 a 2013, el cual pasó de \$24,500 millones de pesos en 2009 , a $\$ 36,500$ en 2010 , subió a $\$ 54,500$ en 2011, se incrementó a $\$ 85,700$ en 2012 y por último pasó a \$121,600 en 2013 (AMIPCI, 2013). 
Los datos disponibles del comercio electrónico también muestran una evolución positiva en esta actividad en los años recientes, como lo señala la Asociación Mexicana de Internet (2016) (Ver Tabla 1.), para el año 2014 el incremento del mismo fue de un 34\% y un 59\% en el año 2015.

Tabla 1. Evolución del Comercio Electrónico en México (miles de millones de pesos)

\begin{tabular}{|l|c|c|c|c|c|c|c|}
\hline & 2009 & $\mathbf{2 0 1 0}$ & $\mathbf{2 0 1 1}$ & $\mathbf{2 0 1 2}$ & $\mathbf{2 0 1 3}$ & $\mathbf{2 0 1 4}$ & $\mathbf{2 0 1 5}$ \\
\hline $\begin{array}{l}\text { Valor estimado del } \\
\text { mercado de comer- } \\
\text { cio electrónico }\end{array}$ & 24.5 & 36.5 & 54.50 & 85.70 & 121.60 & 162.10 & 257.09 \\
\hline
\end{tabular}

Fuente: AMIPCI (2016).

En el estudio de comercio electrónico de la AMIPCI (2016) se observa un crecimiento en las compras realizadas en empresas extranjeras pasando de $57 \%$ en 2015 a 60\% en 2016, siendo Estados Unidos el país de origen de las empresas donde más compran los cibernautas (61\%). En la misma investigación se señala que las tres categorías de productos que más realizan comercio electrónico son ropa y accesorios $(53 \%$ en 2015 y 2016), descargas digitales (49\% en 2015 y 46\% en 2016) y compra de boletos para eventos (35\% en 2015 y $37 \%$ en 2016) (AMIPCI, 2016). Los métodos de pago preferidos para liquidar estas compras son paypal (62\%), tarjetas de crédito (51\%) y tarjetas de débito (56\%).

\section{Metodología}

Con el objetivo de realizar un diagnóstico de las grandes empresas de Iztapalapa que ya cuentan con la posibilidad de realizar transacciones comerciales en línea y así como de conocer el nivel de difusión del comercio electrónico en la demarcación, se hizo una investigación descriptiva que permitió determinar las propiedades, características y perfiles de un conjunto de compañías.

De acuerdo con el INEGi (2013), la Ciudad de México es el área económica más importante del país, ya que además de ser la región con 
un mayor número de unidades económicas establecidas $(8.15 \%)$, es la que contribuye con la mayor proporción de producto interno nacional (16.7\%). Basados en el censo económico de 2014 (INEGI) la delegación Iztapalapa es donde se ubica el mayor número de empresas $(73,321)$, con un personal ocupado de 320,196 empleados y una producción bruta que corresponde al 4.9\% del total de la Ciudad de México.

En este estudio se tomó como unidad de análisis las 273 grandes empresas de esta delegación que ya cuentan con una base tecnológica que les permite implementar el comercio electrónico. La fuente principal de información fueron las empresas que cuentan con 100 o más empleados registradas en el directorio estadístico nacional de unidades económicas del INEGI (2015).

Se llevó a cabo un análisis cuantitativo y de contenido, examinando las páginas de sitios web de las empresas y las aplicaciones de comercio electrónico ya implementadas en las mismas, durante el periodo de mayo de 2017 a septiembre de 2017. El análisis incluyó navegar en todos los niveles del sitio Web y revisar las características de su sitio de comercio electrónico en aquellas empresas que ya lo han adoptado y que funciona como una alternativa de compra-venta.

El análisis de contenido tuvo como primer objetivo determinar el nivel de implementación de la tecnología de internet como base para la adopción de comercio electrónico. Se establecieron variables dicotómicas para determinar la fase en la que se encuentran las grandes empresas de Iztapalapa tomando como referencia la propuesta del modelo de etapas para el desarrollo de esta tecnología de Veciana Vergés y Serrasols i Tarrés (2001). Al llevar a cabo el análisis de contenido se contabilizó la presencia o ausencia de las variables establecidas para cada nivel o fase.

La primera etapa corresponde a la presencia en internet por parte de las empresas a través de la existencia o no de una página web. Asociada con esta presencia inicial en internet se incluyeron variables dicotómicas relacionadas con la inclusión de la organización en directorios en internet de diferente tipo: empresariales, sectoriales y de bolsas de trabajo. Así como variables que dieran cuenta de información general de las 
empresas, por ejemplo: dirección de correo electrónico, dirección física, teléfono, antigüedad, origen de la empresa, grupo al que pertenece la empresa, sitio global, idiomas del sitio, tipo de empresa, posibilidad de establecer contacto personal con la organización, si es empresa socialmente responsable y si cuenta con alguna normalización o certificación.

Para determinar la siguiente fase del desarrollo de la página web se incluyeron las siguientes variables dicotómicas: información general sobre productos o servicios, catálogo de productos o servicios, información específica para clientes, búsqueda de productos o servicios, enlace para clientes, enlace para proveedores, descarga de información, enlace de atención a cliente, enlace para mostrar bolsa de trabajo y enlace para solicitar empleo en la organización.

La última etapa corresponde a la posibilidad de realizar transacciones en la página web, y en nuestro estudio a las empresas que ya han implementado aplicaciones del tipo de intranet, extranet y comercio electrónico. Por lo cual se incluyeron las variables: transacciones en línea, intranet, extranet, comercio electrónico y tipo de comercio electrónico. Debido a que en la última etapa las empresas, a través de su página web, ya permiten realizar una compra en línea, se analizó también si este proceso se lleva a cabo de acuerdo con el modelo multi-etapas para comercio electrónico, el cual incluye la búsqueda e identificación, selección y negociación, facturación, entrega de productos o servicios y servicio posventa. Por lo tanto, se revisaron las siguientes variables: solicitud de cotización, elaboración de cotización en línea, botón de tienda en línea, información y registro del contacto, gestión del contenido, personalización de productos o servicios, modificación de lista de compra, utilización de carrito de compra, orden de compra, forma de pago, método de envío, políticas de devolución, envío de producto, y servicio posventa.

Con la información recopilada y codificada se procedió al análisis estadístico utilizando el software JMP. Se llevó a cabo un análisis descriptivo de frecuencias de distribución y los resultados obtenidos de las variables estudiadas se representaron a través de los porcentajes totales de las mismas. 


\section{Resultados y análisis}

El total de grandes empresas de Iztapalapa registradas en el directorio estadístico nacional de unidades económicas del INEGI (2015) fue de 273. El 57\% pertenecen al sector de manufactura, el 27\% al de comercio y $16 \%$ al de servicio. En esta zona geográfica hay empresas con más de 100 años de antigüedad (9), otras que se fundaron entre 1931 y 1950 (25); se encontró que la mayoría de las organizaciones se establecieron entre 1951 y 1980 (63), 44 unidades económicas se crearon entre 1981 y 1997 y solo 10 empresas se constituyeron a partir del 2001. El 90\% de las unidades económicas son empresas mexicanas y más de la mitad de las mismas (51\%) forman parte de un grupo empresarial. El 86\% de las empresas son mayoristas y el 14\% son empresas minoristas.

Se pudo establecer la base tecnológica de telecomunicaciones relacionada con la adopción de sitios web en las grandes empresas de la delegación Iztapalapa, a través del número de empresas que ya tienen presencia en internet en diferentes etapas. Con respecto al primer nivel de utilización de páginas web informativas de acuerdo con Veciana Vergés y Serrasols i Tarrés (2001) las empresas adoptan esta tecnología con el objetivo de tener presencia en internet e incluyen solamente datos informativos de la mismas (ver Tabla 2).

\section{Tabla 2. Primer nivel de adopción de comercio electrónico: Web informativo}

\begin{tabular}{|l|c|c|c|c|}
\hline \multicolumn{1}{|c|}{ Variable } & $\begin{array}{c}\text { No. de em- } \\
\text { presas que } \\
\text { SI cuentan }\end{array}$ & $\begin{array}{c}\text { No. de } \\
\text { empresas } \\
\text { que NO } \\
\text { cuentan }\end{array}$ & $\begin{array}{c}\text { Porcentaje } \\
\text { de empre- } \\
\text { sas que SI } \\
\text { cuentan }\end{array}$ & $\begin{array}{c}\text { Porcentaje } \\
\text { de empre- } \\
\text { sas que No } \\
\text { cuentan }\end{array}$ \\
\hline Página web & 227 & 46 & $83.15 \%$ & $16.85 \%$ \\
\hline Correo electrónico & 239 & 34 & $87.54 \%$ & $12.45 \%$ \\
\hline $\begin{array}{l}\text { Directorio } \\
\text { empresarial }\end{array}$ & 265 & 8 & $97.97 \%$ & $2.93 \%$ \\
\hline Directorio sectorial & 252 & 21 & $93.30 \%$ & $7.69 \%$ \\
\hline $\begin{array}{l}\text { Directorio de bolsa } \\
\text { de trabajo }\end{array}$ & 250 & 23 & $91.57 \%$ & $8.42 \%$ \\
\hline
\end{tabular}




\begin{tabular}{|l|c|c|c|c|}
\hline Registro visitantes & 75 & 198 & $27.47 \%$ & $72.52 \%$ \\
\hline Sitio global & 61 & 212 & $22.34 \%$ & $77.65 \%$ \\
\hline Otro idioma & 65 & 208 & $23.81 \%$ & $76.19 \%$ \\
\hline $\begin{array}{l}\text { Publicidad de } \\
\text { eventos }\end{array}$ & 112 & 161 & $41.02 \%$ & $58.97 \%$ \\
\hline Establecer contacto & 243 & 30 & $89.01 \%$ & $10.98 \%$ \\
\hline $\begin{array}{l}\text { Certificación o } \\
\text { Normas }\end{array}$ & 160 & 113 & $58.60 \%$ & $41.39 \%$ \\
\hline
\end{tabular}

Fuente: Elaboración propia.

Se encontró que el $88 \%$ de las empresas ya cuentan con una dirección de correo electrónico a diferencia del $83 \%$ de las empresas que tienen un sitio de página web propio, esto puede interpretarse como el primer paso de algunas empresas hacia la digitalización adoptando una dirección de correo electrónico. A las empresas sí les interesa tener una presencia en internet, ya sea a través del desarrollo de su propio sitio o por lo menos formar parte de alguno de los directorios que existen en internet como son empresariales (97\%), sectoriales (92\%), o en referencias para bolsa de trabajo $(92 \%)$.

La mayoría de las páginas web de las empresas no registra la visita de los cibernautas, sólo el 27\% lo hace, perdiendo la oportunidad de llevar un registro de aquellas organizaciones o individuos que regresan y pueden estar interesados en conocer más información sobre algún producto o servicio. Del total de páginas web el $22 \%$ son sitios globales, es decir, muestran la página de la marca, de empresas multinacionales, por lo que la información tiene las mismas características de la empresa y se puede visitar en diferentes países o regiones. Si bien, el 76\% de los sitios, independientemente del origen de las empresas, se pueden desplegar en inglés, probablemente como un elemento para la internacionalización de las empresas, o para fortalecer su presencia a nivel global.

Las empresas en sus sitios en internet presentan información general, como por ejemplo publicidad sobre sus eventos (59\%), proporcionan un contacto (89\%), el cual puede ser a través de correo electrónico (77\%). También indican en su página web si cuentan con alguna certificación 
o cumplen con ciertas normas (59\%). En poco más de la mitad de los casos, se indica si las empresas son socialmente responsables (53\%).

El segundo nivel en el proceso de adopción de comercio electrónico corresponde con un sitio web de procesamiento que permita incluir una serie de elementos o procesos que se pueden llevar a cabo desde el sitio de la empresa (Ver Tabla 3.).

\section{Tabla 3. Segundo nivel de adopción de comercio electrónico: Web de procesamiento}

\begin{tabular}{|l|c|c|c|c|}
\hline \multicolumn{1}{|c|}{ Variables } & $\begin{array}{c}\text { Empresas que } \\
\text { SI incluyen en } \\
\text { su sitio web }\end{array}$ & $\begin{array}{c}\text { Empresas que } \\
\text { No incluyen } \\
\text { en su sitio } \\
\text { web }\end{array}$ & $\begin{array}{c}\text { Porcentaje de } \\
\text { empresas que } \\
\text { SI incluyen en } \\
\text { su sitio web }\end{array}$ & $\begin{array}{c}\text { Porcentaje de } \\
\text { empresas que } \\
\text { NO incluyen } \\
\text { en su sitio web }\end{array}$ \\
\hline $\begin{array}{l}\text { Descripción de } \\
\text { productos o servicios }\end{array}$ & 232 & 40 & $85.29 \%$ & $14.70 \%$ \\
\hline Catálogo & 192 & 81 & $70.33 \%$ & $29.67 \%$ \\
\hline Búsquedas & 153 & 120 & $56.04 \%$ & $43.95 \%$ \\
\hline Liga clientes & 144 & 129 & $52.74 \%$ & $47.25 \%$ \\
\hline Liga proveedores & 79 & 194 & $28.93 \%$ & $71.06 \%$ \\
\hline Liga público & 85 & 188 & $31.13 \%$ & $68.86 \%$ \\
\hline Aplicaciones & 79 & 194 & $28.93 \%$ & $71.06 \%$ \\
\hline Liga atención cliente & 150 & 123 & $54.94 \%$ & $45.05 \%$ \\
\hline Bolsa de trabajo & 42 & 231 & $15.38 \%$ & $84.61 \%$ \\
\hline Solicitud de empleo & 40 & 233 & $14.65 \%$ & $85.34 \%$ \\
\hline
\end{tabular}

Fuente: Elaboración propia.

En la mayoría de los sitios ya se incluye la descripción de productos o servicios (85\%), así como el catálogo (70\%) de sus mercancías. También es posible que en la página el visitante pueda realizar búsquedas específicas de productos (56\%), que se presenten comunicaciones dirigidas a los clientes $(69 \%)$, o en su defecto enlaces para clientes $(53 \%)$, así como enlaces para proveedores $(29 \%)$ o para público en general (31\%). En el mismo sentido, pero en un menor porcentaje de empresas, es posible que el usuario pueda descargar aplicaciones, manuales técnicos o documentos complementarios a los productos o servicios 
(29\%). Los sitios también cuentan con un enlace específico de atención al cliente (55\%). Otro proceso que las empresas ya están incluyendo en sus páginas web es el que corresponde a la publicidad de su bolsa de trabajo (15\%) y en algunos casos, incluso, la posibilidad de enviar una solicitud de empleo en línea (15\%).

En esta fase, se aprecia que las empresas empiezan a establecer herramientas o medios para tener una comunicación bidireccional con los visitantes de su página, de tal forma que pueda existir un canal de comunicación para obtener, en particular, retroalimentación de sus clientes actuales o clientes potenciales. Además, les facilita el proceso de búsqueda y selección de un producto o servicio, ya sea para su compra tradicional o por lo menos para hacer una cotización en línea que les facilite el proceso de compra. Sin embargo, las empresas han continuado trabajando con sus sitios web de procesamiento pues no todos los interesados efectúan sus pedidos o cotizaciones a través de la página de internet.

El tercer nivel de un sitio correspondería con la característica de ser transaccional, es decir en esta etapa la empresa ya incorpora procesos para poder llevar a cabo transacciones en su sitio (Ver Tabla 4.).

\section{Tabla 4. Tercer nivel de adopción de comercio electrónico: Web transaccional}

\begin{tabular}{|l|c|c|c|c|}
\hline \multicolumn{1}{|c|}{ Variables } & $\begin{array}{c}\text { Empresas } \\
\text { que SI } \\
\text { incluyen }\end{array}$ & $\begin{array}{c}\text { Empresas } \\
\text { que NO } \\
\text { incluyen }\end{array}$ & $\begin{array}{c}\text { Porcentaje de } \\
\text { empresas que } \\
\text { SI incluyen }\end{array}$ & $\begin{array}{c}\text { Porcentaje de } \\
\text { empresas que } \\
\text { NO incluyen }\end{array}$ \\
\hline Transacciones & 56 & 217 & $20.51 \%$ & $79.48 \%$ \\
\hline Intranet & 93 & 180 & $34.06 \%$ & $65.93 \%$ \\
\hline Extranet & 69 & 204 & $25.27 \%$ & $74.72 \%$ \\
\hline $\begin{array}{l}\text { Comercio } \\
\text { electrónico }\end{array}$ & 53 & 220 & $19.41 \%$ & $80.58 \%$ \\
\hline
\end{tabular}

Fuente: Elaboración propia.

En el tercer nivel de adopción de comercio electrónico, el 21\% de los sitios de las empresas incluyen la posibilidad de realizar, desde la 
misma, transacciones electrónicas; en algunos casos, las páginas incluyen aplicaciones que implican otro nivel de funcionamiento como la implementación de intranet (34\%) y extranet (25\%).

En este nivel se encontró que 53 empresas ya han adoptado el comercio electrónico, lo cual representa el 19\% de la población de grandes empresas de Iztapalapa. El tipo de comercio electrónico que realizan es entre empresas, es decir comercio B2B (84\%) y con consumidores o comercio B2C (26\%) (O’Brien y Marakas, 2007; Stair y Reynolds, 2006).

De este grupo de empresas se encontró que el 51\% se ubican en el sector industrial, $41 \%$ en el comercial y $8 \%$ en el sector servicios. Con respecto a la antigüedad de las empresas 7 de las organizaciones con más de 100 años de operación ya han incorporado el comercio electrónico en sus sitios de internet. De los sitios de internet que incluyen el año de creación de las empresas se encontró que dos empresas se establecieron antes del 1950, 17 empresas fundadas entre 1951 y 1980 ya han adoptado está tecnología y solamente 7 empresas creadas entre 1981 y 1997 también la utilizan. Llama la atención que ninguna de las empresas más jóvenes creadas después del año 2001 (10) haya implementado aún el comercio electrónico. Al analizar la antigüedad de las empresas se muestra que aquellas que ya tienen experiencia, están consolidadas y tienen una presencia de marca les resulta más fácil dar el salto a la oferta de sus productos y servicios en línea que a empresas de reciente creación, ya que ninguna de las empresas creadas a partir del año 2000 han adoptado el comercio electrónico.

De este grupo de empresas, el 92\% son mexicanas y el 19\% opera en internet a través de un sitio global. Casi la mitad de las mismas forma parte de un grupo empresarial (47\%), con lo cual se aseguran que las operaciones de compra venta en línea sean uniformes para todas las empresas de la multinacional.

Como lo señala Sojo Obando (2001), la adopción del comercio electrónico implica una inversión importante tanto en hardware como en software, por lo que resulta más fácil para aquellas empresas que ya cuentan con una base tecnológica, así como en el caso de la experien- 
cia en uso de innovaciones tecnológicas posibilita adoptar el comercio electrónico. Lo cual se muestra en el conjunto de empresas estudiadas que ya utilizan esta tecnología, pues, de igual forma se han implementado otras innovaciones basadas en la plataforma de internet, como el empleo de intranet $(80 \%)$ y de extranet $(74 \%)$.

Las herramientas mencionadas son complementos necesarios para el uso del comercio electrónico como se muestra en el modelo multi-etapas el cual señala la importancia de la interconexión y comunicación entre diferentes áreas de la empresa que están involucradas en los procesos de compraventa (O'Brien y Marakas, 2007; Stair y Reynolds, 2006). Así mismo, seguramente las empresas que forman parte de un grupo empresarial (47\%) les será más fácil compartir y adoptar de forma más viable estas aplicaciones.

Como parte de un sistema de comercio electrónico se requiere que las organizaciones además de contar con una plataforma de internet hayan implementado un modelo multi-etapas para comercio electrónico con el fin de que el usuario pueda realizar el proceso de ciclo de vida de ventas a través de compra en línea, de manera eficiente y segura.

El primer elemento con el que deben contar los sitios de comercio electrónico de las empresas es un control de acceso y seguridad (O’Brien y Marakas, 2007; Stair y Reynolds, 2006). El 83\% de las empresas requieren de un registro de usuario y el 79\% solicitan información del visitante. A partir de esta información la empresa irá construyendo el perfil del cliente y personalizando el sitio para cada uno de ellos. Este primer paso implica una tarea relevante para poder recopilar datos de sus compradores, analizar su comportamiento y hacerle recomendaciones en sus futuras visitas con base en su perfil y gustos (Sojo Obando, 2001); por lo cual resulta muy importante contar con esos datos e implica una omisión importante para las empresas que no lo registran. Un pequeño porcentaje de empresas $(8 \%)$ no cuenta con las herramientas adecuadas para poder ejecutar correctamente la compra venta en línea, ya que, por ejemplo, no tiene una liga directa para iniciar el proceso de compra o no tiene la posibilidad de registrar a los clientes (17\%) o tampoco piden información del usuario (21\%). Estas deficiencias ocasio- 
nan que los clientes potenciales desconfíen al decidirse para establecer relaciones con la empresa.

Para que el cliente pueda seleccionar el producto o servicio que desea, así como el precio de la compra, es necesario que la página tenga una herramienta para gestionar el contenido de sus productos o servicios, de tal forma que el usuario pueda buscar aquello que le interese, ver las características generales, así como los detalles de los mismos $\mathrm{y}$, de ser necesario, modificar la selección que previamente se hubiera realizado. El 91\% de las empresas cuenta con un gestor de contenido y catálogo para que el visitante pueda tener mayor certeza en el producto que se va adquirir. El $58 \%$ de estos sitios permite al cliente poder personalizar su producto, aprovechando uno de los beneficios de la utilización de esta aplicación (Sojo Obando, 2001). Y una vez que lo desea agregarlo al carrito de compra (96\%) (la mayoría de las empresas han utilizado el símil de utilizar un carrito de compra como se hace en las compras en las tiendas de autoservicio). De igual forma en 91\% de los sitios, el visitante podría aprovechar de una de las ventajas del empleo de esta tecnología: modificar su compra y actualizarla antes de formalizarla (O’Brien y Marakas, 2007; Stair y Reynolds, 2006).

A continuación, el sistema necesita incluir todo lo relacionado con la administración de pago, cuando el cliente está decidido a llevar a cabo la compra, en el $96 \%$ de los sitios se genera una orden de compra y se selecciona el método de pago para facturar la transacción. En el 97\% de los sitios el pago es en línea, ya sea con tarjeta de crédito o de débito (42\%) o a través de paypal (55\%). Es decir, los datos correctos y generación de documentos y operaciones monetarias se realizan de forma correcta a través de las diferentes áreas de la empresa (O'Brien y Marakas, 2007; Stair y Reynolds, 2006). El uso de métodos seguros de pago, como tarjeta de crédito o débito o paypal, permite a los clientes tener el respaldo de una institución financiera al ejecutar la compra o hacer alguna reclamación (O’Brien y Marakas, 2007; Stair y Reynolds, 2006).

Una de las ventajas del comercio electrónico es que evita que el cliente se tenga que desplazar para obtener su producto (Sojo Obando, 2001), por ello, una parte de las etapas del proceso debe determinar el 
método de entrega o envío del producto, en ese sentido el 74\% de las empresas lo hace a través de mensajería y el $26 \%$ directamente en sus locales. Otro resultado destacado de la investigación es que la mayoría de las unidades económicas (94\%) solo practican un comercio electrónico nacional, mientras que el 11\% sí efectúa entregas en el extranjero, contrario a una de las posibles ventajas de esta herramienta que les permite a las empresas tener una presencia global (Sojo Obando, 2001). Puede ser que debido a los costos de mensajería o a las características del producto o servicios de las empresas todavía no les convenga financieramente su internacionalización. O también a las restricciones comerciales existentes entre diferentes países.

En las transacciones de comercio electrónico un elemento importante es la posibilidad de devolver el producto, por eso el $62 \%$ de las empresas sí cuenta con políticas de devolución, contrario a un 38\% de las empresas que no tiene políticas de devolución, lo cual no es un incentivo para fomentar el uso de estas tecnologías.

La última etapa corresponde a la existencia de un servicio posventa que permita al cliente interactuar con la organización, estableciendo la posibilidad de un canal de comunicación con la empresa (Sojo Obando, 2001). Esta fase puede resultar un elemento diferenciador con respecto a las ventas tradicionales y el $62 \%$ de las organizaciones en línea sí lo incluyen en su página web. Finalmente, una de las particularidades más relevantes en el desarrollo de esta tecnología es la seguridad en la transacción comercial, la cual no la presentan el 20\% de las empresas, desincentivando la posibilidad de atraer clientes y fomentando la idea de que el comercio electrónico sigue siendo una práctica insegura (Fernández, Hurtado y Peral, 2005). Este es un elemento que seguramente limita el comercio electrónico ya que cada vez más los compradores ponen atención en el elemento de seguridad en las transacciones.

Los resultados indican que, a pesar del aumento en adopción y difusión del comercio electrónico por parte de las empresas tanto a nivel mundial como nacional, su implementación todavía es baja en la región de estudio: únicamente el 19\% de las grandes empresas en Iztapalapa están utilizando esta tecnología. 
Siendo una característica de la aplicación de comercio electrónico su universalidad y estandarización le permite su implementación en cualquier tipo de organización (O’Brien y Marakas, 2007; Stair y Reynolds, 2006); se encontró que esta tecnología se utiliza en unidades económicas de los tres sectores, prácticamente ya lo emplean la mitad (51\%) de las empresas del sector industrial, casi la mitad de empresas comerciales $(42 \%)$ y muy pocas empresas del sector servicios (8\%). Es evidente que para las empresas comerciales esta aplicación es un paso natural el trasladar sus operaciones al comercio electrónico.

Casi todos los sitios de internet analizados (91\%) permiten realizar búsquedas de productos, revisar sus características y seleccionar lo que desean a través de la utilización de un carrito de compra (96\%). De igual forma más de la mitad de las empresas cuenta con la opción de personalizar la compra (58\%).

\section{Conclusiones}

Los resultados obtenidos permitieron determinar el nivel de desarrollo de páginas web de un conjunto de grandes empresas de Iztapalapa como parte de la adopción de comercio electrónico en esta zona de la Ciudad de México. Aunque el $83 \%$ de estas unidades económicas ya tiene un sitio en Internet de tipo informativo, las empresas todavía deben seguir desarrollándose para pasar a las etapas de web de procesamiento y web transaccional. En la medida en que las organizaciones sigan desarrollando sus sitios web lograrán incluir nueva información y más procesamientos, como base para la adopción de comercio electrónico.

A través de este estudio, se obtuvo un panorama de la difusión de tecnologías de información en un grupo de empresas, y en particular del comercio electrónico, el cual evidencia un nivel de difusión bajo, puesto que esta aplicación no se ha adoptado ni aprovechado lo suficiente a pesar de sus múltiples beneficios. Aunque la difusión del comercio electrónico en México ha crecido de acuerdo con las estadísticas (AMIPCI, 2013, 2016), su difusión todavía resulta incipiente ya que solamente el 19\% de grandes empresas de Iztapalapa lo han adoptado. Incluso en 
algunos casos la aplicación descuidada del comercio electrónico por parte de algunas empresas que no han incluido medidas de seguridad adecuadas, puede provocar que los clientes potenciales no establezcan relaciones comerciales con la empresa.

Para aquellas unidades económicas que están utilizado el comercio electrónico, ya se encuentran aprovechando las ventajas vinculadas con la creación de nuevos canales de mercadotecnia y ventas, la posibilidad de acceder de forma interactiva a catálogos, listas de precios, la alternativa de personalizar los productos de acuerdo a las necesidades de los clientes y contar con un servicio al cliente posventa (Ríos Ruíz, 2014).

Así mismo, el comercio electrónico representa para las empresas una disponibilidad ilimitada de horarios, presencia permanente en el ciberespacio y, por lo tanto, una mayor competitividad, eliminación de intermediarios y un incremento en el conocimiento del cliente (Ríos Ruíz, 2014).

Es importante que antes de tomar la decisión de incursionar en el comercio electrónico las empresas consideren los siguientes aspectos: cuál es el objetivo estratégico que motiva la incursión en el comercio electrónico, es importante determinar su política de precios, hay que afianzar todos los aspectos de seguridad (en el pago, en la entrega, en el producto), conocer el perfil del cliente, establecer una estrategia de comunicación (presencia en buscadores y portales importantes, Links y banner, anuncios publicitarios en periódicos, revistas especializadas, radio, televisión, mercadeo personalizado, vallas publicitarias, promociones), desarrollar el sitio (reforzar la estrategia de comercio electrónico con personal propio de la empresa, contratar a terceros para su diseño e implementación) distribución (venta de productos por Internet a través de intermediarios, inversión a futuro) (Sojo Obando, 2001).

En la segunda etapa del estudio, mediante la aplicación de una encuesta con las grandes empresas de Iztapalapa, se podrá analizar si existen otras ventajas asociadas con la reducción de costos, la disminución de tiempo en procesos, mejor servicio al cliente, reducción de inventarios y una mejor coordinación entre las empresas involucradas en el proceso de compra-venta. 


\section{Bibliografía}

AMIPCI, E-commerce study in Mexico, Asociación Mexicana de Internet, AMIPCI, A.C. , México, 2016.

AMIPCI, Estudio de Comercio Electrónico México 2013, Asociación Mexicana de Internet, México, 2013.

Daniel, E., Wilson, H. y Myers, A., “Adoption of e-commerce by SMEs in the UK: towards a stage model", International Small Business Journal, 20 (3), 2002, pp. 253-70.

Fernandez Nodarse, Francisco A., "Sobre Comercio electrónico en la WEB 2.0 y 3.0", Revista Cubana de Ciencias Informáticas, 2013, 7, Septiembre. Disponible en: http://www.redalyc.org/articulo. oa?id=378334198009> [Consultado 16 de agosto de 2017].

Fernández, Ma. del Rosario, Hurtado, Ma Antonia y Peral, Diego, “Comercio Electrónico en la Era Digital”, España Razón y Palabra, 10 (45), junio-julio, Instituto Tecnológico y de Estudios Superiores de Monterrey, 2005.

Fernández-Portillo, Antonio, et al., "La importancia de la Innovación en el Comercio Electrónico", Universia Business Review, (Julio-Septiembre). Disponible en: http://www.redalyc.org/articulo. oa? id=43341001006 [Consultado 14 de agosto de 2017].

Frasquet Deltoro, Marta, Mollá Descals, Alejandro y Ruiz Molina, María Eugenia, Factores determinantes y consecuencias de la adopción del comercio electrónico B2C: una comparativa internacional Estudios Gerenciales, 2012, 28 (Abril-Junio). Disponible en:http:// www.redalyc.org/articulo.oa?id=21224852006 [consultado 16 de agosto de 2017].

Fredriksson, Torbjörn. "E-commerce and Development, Key Trends and Issues", UNCTAD WSIS Forum 2013, WTO, Geneva, 14 May 2013, p. 13.

Guerrero Cuéllar, Rubén y Rivas Tovar, Luis Arturo, “Comercio electrónico en México: propuesta de un modelo conceptual aplicado a las PyMEs", Revista Internacional de Ciencias Sociales y Huma- 
nidades, SOCIOTAM, XV (enero-junio). Disponible en: http://www. redalyc.org/articulo.oa?id $=65415104$ [consultado 16 de agosto de 2017].

INEGI, Encuesta Nacional sobre Disponibilidad y Uso de Tecnologías de la Información en los Hogares (ENDUTIH), Instituto Nacional de Estadística, Geografía e Informática, México, 2013.

INEGI, Censos Económicos 2014. Instituto Nacional de Estadística y Geografía, México. Disponible en: http://www3.inegi.org.mx/sistemas/cce2014/ [Consultado 14 de junio de 2017].

INEGI, Directorio Estadístico Nacional de Unidades Económicas. Instituto Nacional de Estadística y Geografía. Disponible en: http:// www.beta.inegi.org.mx/app/mapa/denue/ [Consultado 14 de junio de 2017].

INEGI, Usuarios de Tecnologías de Información 2001-2015. Instituto Nacional de Estadística y Geografia. Disponible en: http://www. inegi.org.mx [Consultado 17 de mayo de 2016].

Montijano Guardia, Francisco, Bernal Jurado, Enrique, Del Águila Obra, Ana R. y Padilla Meléndez Antonio, "Comercio electrónico y servicios financieros. Impacto sobre el sector asegurador en España”, Economía Industrial, No. 334. 2002-II, pp. 45-156 Disponible en: https://www.researchgate.net/publication/28061278 [consultado 17 de agosto de 2017].

O'Brien, J. A. y Marakas, G. M., Introduction to Information Systems, 13a. ed., McGraw-Hill Irwin, New York, 2007, p. 543.

Pecoy Taque, Martin, Delito en el Comercio Electrónico Prisma Jurídico, 2011, p. 10 (Enero-Junio). Disponible en: http://www.redalyc. org/articulo.oa?id=93420939012 [consultado 14 de agosto de 2017].

Ríos Ruiz, Alma de los Ángeles, “Análisis y perspectivas del comercio electrónico en México", Revista Venezolana de Información, Tecnología y Conocimiento, (Septiembre-Diciembre). Disponible en: http://www.redalyc.org/articulo.oa?id=82332932006> ISSN 16907515 [consultado 16 de agosto de 2017]. 
Rodríguez-Ardura, I., Meseguer Artola, A., y Vilaseca i Requena, J., "El Comercio Electrónico en Perspectiva: Dinámica y Desencadenantes", Investigaciones Europeas de Dirección y Economía de la Empresa, p. 14 (Septiembre-Diciembre). Disponible en: http:// www.redalyc.org/articulo.oa?id=274120249003 [consultado 16 de agosto de 2017].

Sepúlveda Robles, Daniel Eduardo, Sepúlveda Jiménez, Daniel, Pérez Soto, Francisco, Figueroa Hernández, Esther, "Modelo para la adopción del comercio electrónico en el sector agroindustrial mexicano", Análisis Económico, XXXI (Enero-Abril), 2016. Disponible en: http://buap.redalyc.org/articulo.oa?id=41344590004 [consultado 16 de agosto de 2017].

Stair, R. M. y Reynolds, G. W., Principles of Information Systems. A Managerial Approach, Thompson, United States, 2006, p. 768.

Sojo Obando, Saray, "Comercio electrónico en Costa Rica, InterSedes", Revista de las Sedes Regionales, p. 2. Disponible en: http:// www.redalyc.org/articulo.oa? $\mathrm{id}=66620313$ [consultado 11 de agosto de 2017].

UNCTAD, Maximizing the development gains from e-commerce and the digital economy, Trade and Development Board Intergovernmental Group of Experts on E-commerce and the Digital Economy, Julio 2017, p. 21. Disponible en: www.unctad.org/meetings/en/SessionalDocuments/tdb_edeld2.en.pdf [consultado 11 de Agosto 2017].

Veciana Vergés, José María y Christian, Serrasols i Tarrés, "La economía digital en España: un Análisis Prospectivo", en Del Aguila, Ana Rosa y Antonio Padilla (coords.), E-Business y Comercio Electrónico. Un enfoque estratégico, Ediciones Ra-Ma, Madrid, 2001, pp. 41-58. 